\title{
A Case Treated With Photoimmunotherapy Under a Navigation System for Recurrent Lesions of the Lateral Pterygoid Muscle
}

\author{
ISAKU OKAMOTO, TAKURO OKADA, KUNIHIKO TOKASHIKI and KIYOAKI TSUKAHARA \\ Department of Otorhinolaryngology, Head and Neck Surgery, Tokyo Medical University, Tokyo, Japan
}

\begin{abstract}
Background: Head and neck photoimmunotherapy specifically destroys tumor cells by irradiating them with 690 $n m$ red light after administering cetuximab sarotalocan sodium. The key point in the technique of photoimmunotherapy is to set the irradiation area precisely with a margin added to the target lesion while securing a safety zone with large vessels such as the internal carotid artery. Case Report: At our Institution, we performed photoimmunotherapy in combination with use of a navigation system on a male patient in his 70s with maxillary gingival carcinoma who had a recurrent lesion deep in the lateral pterygoid muscle. Three months after photoimmunotherapy, the superficial lesion had disappeared, the area of contrast within the lateral pterygoid muscle had improved, and the patient was deemed to have a complete response. Conclusion: The Navigation System was successfully used in combination with photoimmunotherapy. Additionally, this allowed the location of the internal carotid artery to be confirmed in real time.
\end{abstract}

Head and neck photoimmunotherapy specifically destroys tumor cells by irradiating them with $690 \mathrm{~nm}$ red light after administration of cetuximab sarotalocan sodium $(1,2)$. Hisataka Kobayashi et al. at the National Cancer Institute in the U.S. developed this therapy (3), and it was approved in Japan in September 2020 for the treatment of unresectable recurrent head and neck cancer and became available in January 2021.

This article is freely accessible online.

Correspondence to: Isaku Okamoto, MD, Ph.D., Department of Otorhinolaryngology, Head and Neck Surgery, Tokyo Medical University, 6-7-1 Nishishinjuku, Shinjuku-ku, Tokyo 160-0023, Japan. Tel: +81 333426111, Fax: +81 333469275, e-mail: isaku@tokyo-med.ac.jp

Key Words: Photoimmunotherapy, navigation system, head and neck carcinoma, maxillary gingival carcinoma.
The key point regarding the technique of photoimmunotherapy is to accurately set the irradiation area with a margin added to the target lesion while ensuring a safety zone with large vessels such as the internal carotid artery. The setting before irradiation has a significant impact on treatment results. Laser irradiation is performed using two types of devices: a frontal diffuser for superficial lesions and a cylindrical diffuser for deep lesions (4). In the case of superficial lesions, the pre-irradiation setting is relatively easy. However, in the case of deep lesions, imaging such as ultrasound or computed tomography (CT) that can depict the lesion in real time is useful to understand the lesion extension plus margins. If the target lesion is in the neck, ultrasound should be used. However, there are areas such as the oral cavity, nasal cavity, and paranasal sinuses, where it is difficult to employ an ultrasound probe. For lesions in these areas, combination with a Navigation System is useful. At our Institution, we performed photoimmunotherapy using a Navigation System on a lesion that recurred in the deep part of the lateral pterygoid muscle after treatment for maxillary gingival carcinoma.

\section{Case Report}

This case report describes a male patient in his 70s with left maxillary gingival carcinoma (T4aN2bM0 stage IVA, squamous cell carcinoma). Written informed consent was obtained from the patient for treatment, inclusion in medical research, and publication of medical data.

The TNM classification of the tumor was determined according to the Union for International Cancer Control version 7 criteria (5). The patient underwent a partial left maxillectomy plus reconstruction with a forearm flap. Histopathological examination revealed a positive deep transection of the lateral pterygoid muscle, and the patient underwent postoperative cisplatin and radiation therapy (60 Gy). Within 6 months of the last dose of cisplatin, a superficial lesion appeared on the posterior wall of the left maxillary sinus, and a biopsy diagnosed squamous cell carcinoma. The patient was treated with nivolumab for locally advanced head and neck 
A

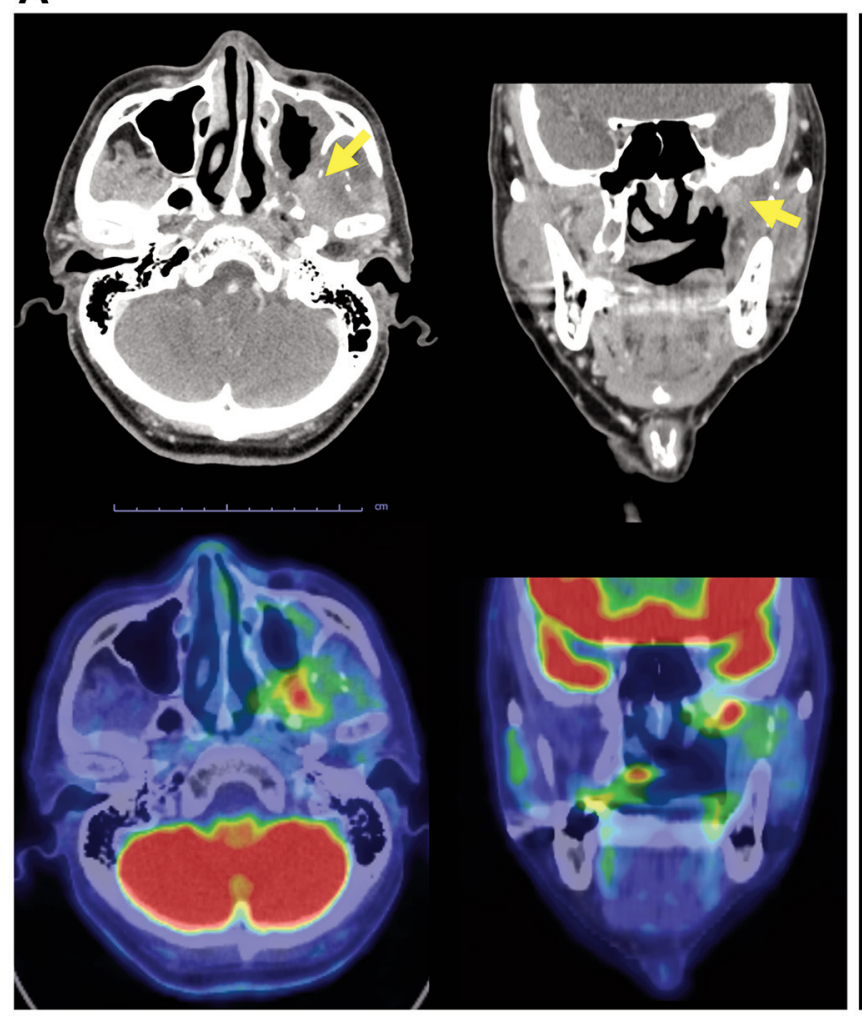

B

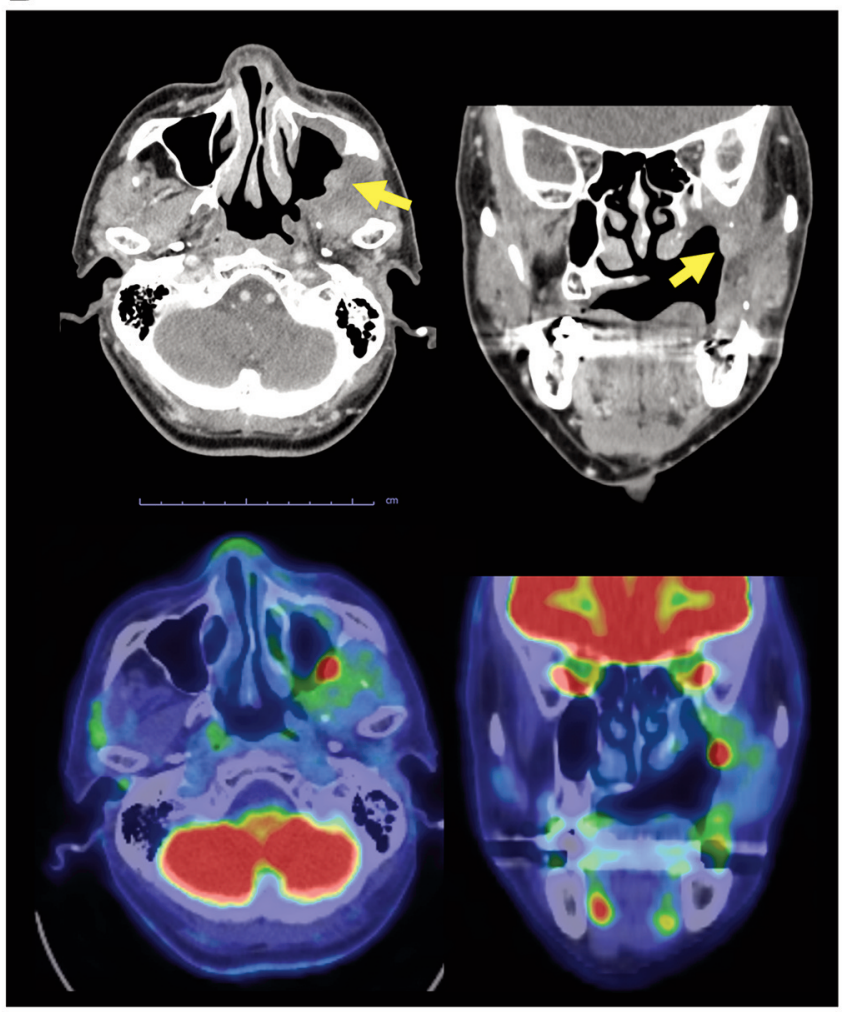

Figure 1. Computed tomography (upper panels) and positron-emission tomography-CT (lower panels) of the target lesion (yellow arrows) in axial and coronal planes before photoimmunotherapy. A: The lesion extended from the periphery of the lateral pterygoid muscle to the base of the skull, $8 \times 18 \mathrm{~mm}$, with a maximum standard uptake value=13.03. B: The superficial lesion on the posterior wall of the maxillary sinus was $10 \times 8$ mm.

cancer. Nivolumab reduced the size of the tumor but, 6 months later, superficial lesions on the posterior wall of the maxillary sinus reappeared. Since the tumor recurred in the reconstructed area, radical resection was difficult and photoimmunotherapy was considered. The patient was informed about photoimmunotherapy, and consent was obtained.

Endoscopic findings showed superficial lesions with redness and elevation at the margins of the reconstruction. Cervical contrast-enhanced CT and positron-emission tomography-CT showed a lesion extending from the periphery of the lateral pterygoid muscle to the base of the skull (Figure 1A) and a superficial lesion on the posterior wall of the maxillary sinus (Figure 1B), which were designated as the target lesions.

Drug administration on the day before photoimmunotherapy and surgery. Cetuximab sarotalocan sodium $\left(640 \mathrm{mg} / \mathrm{m}^{2}\right)$ was administered intravenously over $2 \mathrm{~h}$.

Photoimmunotherapy session. For laser treatment, we used a photodynamic therapy semiconductor laser (BioBrade ${ }^{\circledR}$ laser; Rakuten Medical, Tokyo, Japan) and photodynamic therapy semiconductor laser probes (BioBrade ${ }^{\circledR}$ cylindrical diffuser, BioBrade ${ }^{\circledR}$ frontal diffuser, and BioBrade ${ }^{\circledR}$ needle catheter; Rakuten Medical, Tokyo, Japan). A cylindrical diffuser, emitting a cylindrical laser beam with a radius of $10 \mathrm{~mm}$, was used for intra-tissue irradiation (4). The FusionTM ENT Navigation System (Medtronic, Sunnyvale, CA, USA) was employed, which uses a magnetic field to recognize the positional relationship between the patient and surgical instruments. While displaying the CT in three directions, it is possible to check tumor position and extension with the pointer, with an error within $1 \mathrm{~mm}$. Compared to the optical navigation system, the set-up time is also shorter (6). An ENFVH2 otolaryngology video scope (Olympus, Tokyo, Japan) was used to secure the surgical field in the oral cavity.

After induction of general anesthesia, first, the mucosal surface of the target lesion was measured over two areas (i) $7 \times 7 \mathrm{~mm}$ and (ii) $9 \times 12 \mathrm{~mm}$ (Figure 2A). Next, the direction of the deep infiltration of the lateral pterygoid muscle and the absence of the internal carotid artery in the vicinity were confirmed using the pointer of the Navigation System (Figure 2B). A $50-\mathrm{mm}$ needle catheter was inserted in the same 

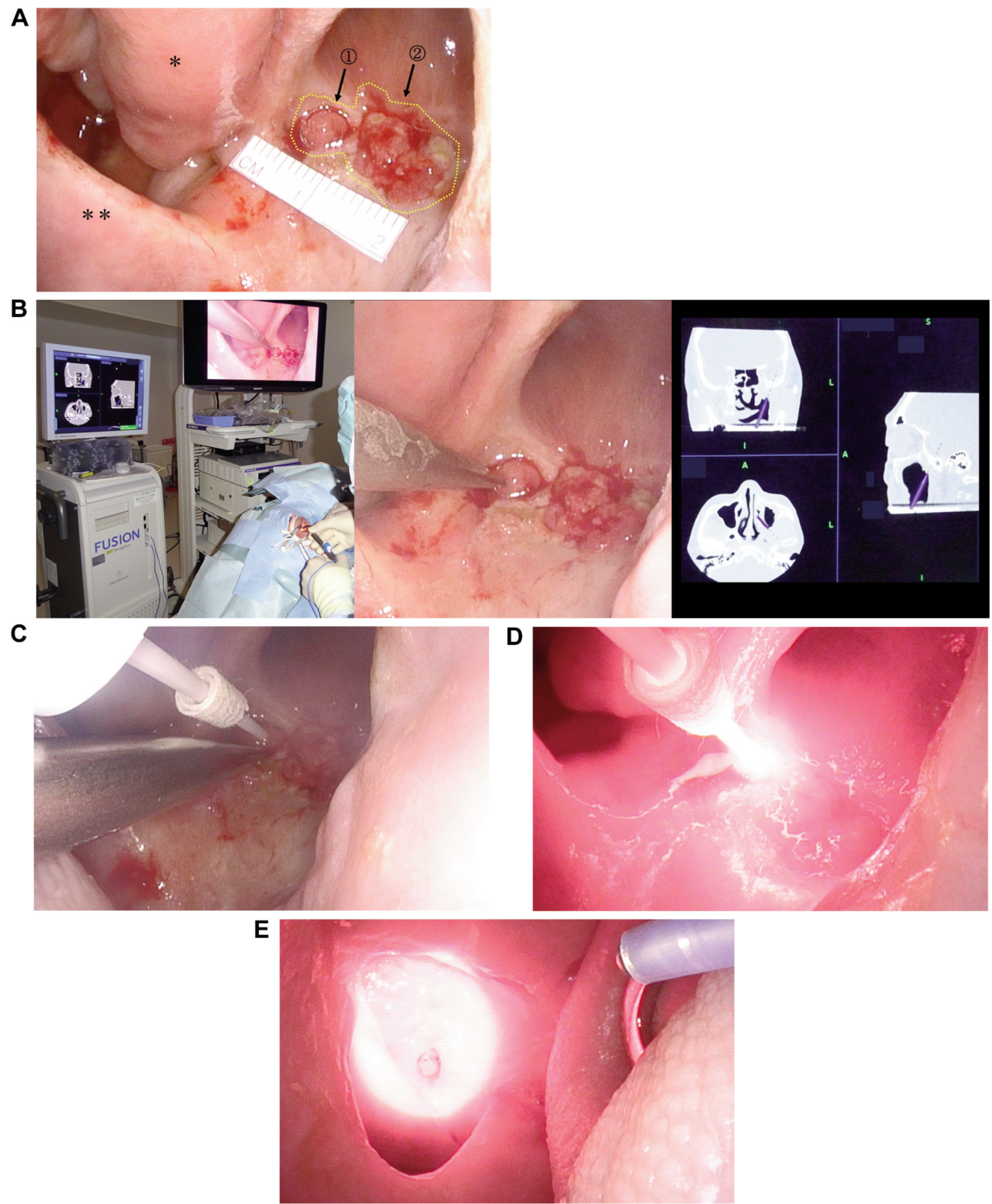

Figure 2. Photoimmunotherapy session and course of treatment. The mucosal surface of the target lesions was delineated and measured (1) $7 \times 7$ $\mathrm{mm}$, (2) $9 \times 12 \mathrm{~mm}) *$ Left inferior turbinate; **hard palate. B: The pointer of the Navigation System, confirming the direction of the deep lesion in the lateral pterygoid muscle. C: Puncturing by the needle catheter in the same direction as the pointer. D: Laser irradiation using a cylindrical diffuser. E: Laser irradiation using a frontal diffuser. 


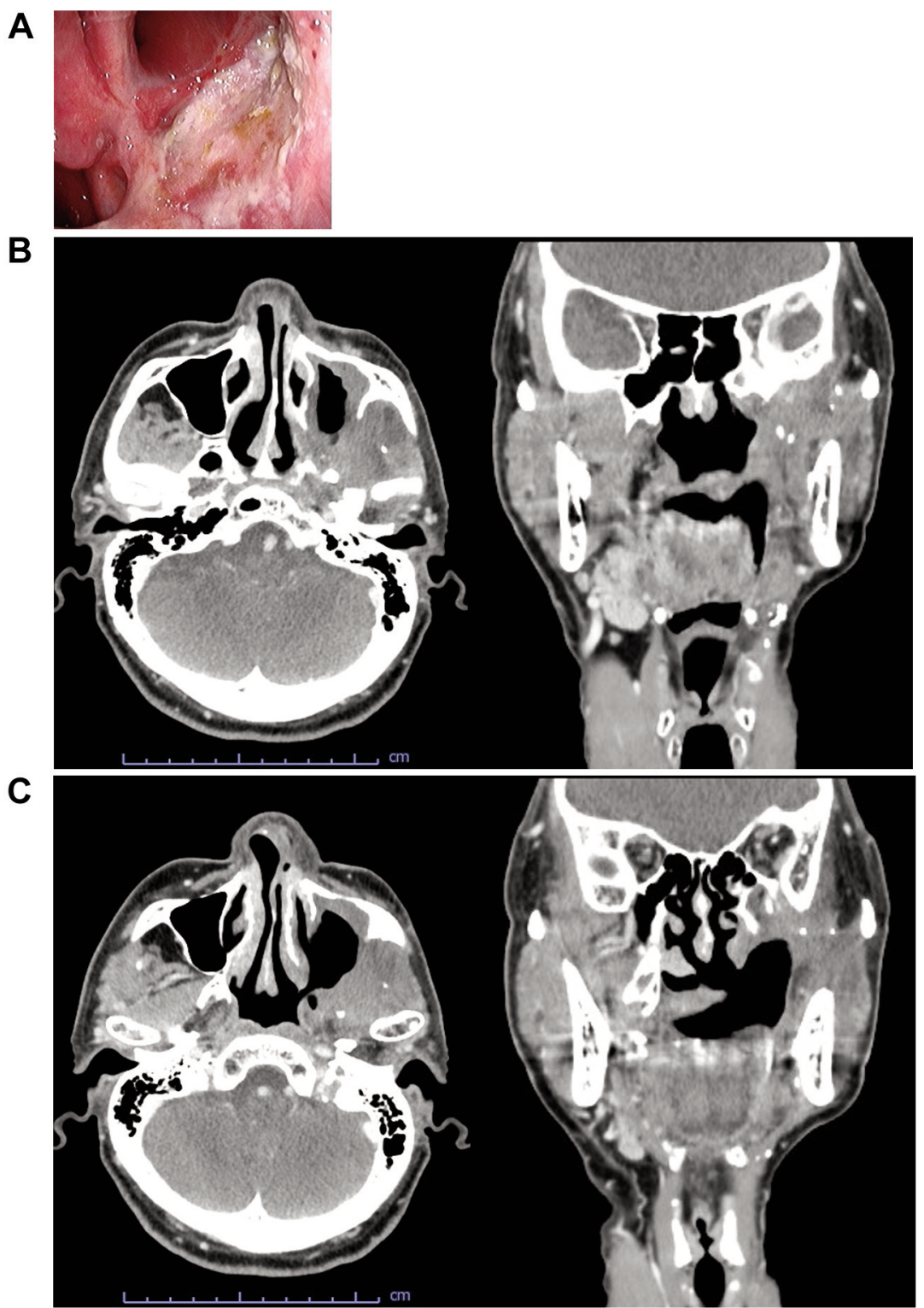

Figure 3. Target lesion after photoimmunotherapy. A: Endoscopic findings. B: Computed tomography (CT) (axial and coronal planes) of a lesion extending from the periphery of the lateral pterygoid muscle to the base of the skull. C: CT (axial and coronal plane) of a superficial lesion on the posterior wall of the maxillary sinus.

direction as the pointer (Figure 2C); $20 \mathrm{~mm}$ from the tumor surface, the catheter hit the skull base bone and was fixed. A cylindrical diffuser of $30 \mathrm{~mm}$ was inserted into the needle catheter, and the first irradiation was performed (Figure 2D). The superficial lesions were then treated. There were two superficial lesions in the vicinity, and their combined size was about $20 \mathrm{~mm}$. Therefore, including the margin, the irradiation diameter of the frontal diffuser was set to $38 \mathrm{~mm}$, and irradiation was performed (Figure 2E). The total irradiation time was 9 min and $43 \mathrm{~s}$, and the total surgery time was 55 min from the start of measurement to the end of surgery.
Endoscopic findings (Figure 3A) and CT (Figure 3B and C) at 3 months after photoimmunotherapy are shown. The superficial lesion had disappeared and the area of contrast within the lateral pterygoid muscle had improved. The patient was judged to be in complete response.

\section{Discussion}

We performed photoimmunotherapy in combination with use of a navigation system for a lesion that recurred in the deep part of the lateral pterygoid muscle after reconstructive 
treatment of maxillary gingival carcinoma. To the best of our knowledge, this is the first case in which combined use of a navigation system with photoimmunotherapy has been reported to be effective.

The wavelength of the red light used in photoimmunotherapy is $690 \mathrm{~nm}$. We used an antibody conjugated with IR700, a photo-activatable dye that absorbs red light. The antibody-IR700 complex binds to the target molecule on the cancer cell membrane, and the cell membrane is damaged when irradiated with near-infrared light (7). The ionic balance of the cell membrane is disrupted, and extracellular fluid flows into the cell, causing it to expand Eventually, cell rupture and cell death occur (8). In addition, necrotic tumour cells release cell death signals (damageassociated molecular patterns), such as adenosine triphosphate, calreticulin, and high mobility group box 1. Cancer antigens and damage-associated molecular patterns activate the immune system, leading to immunogenic cell death, in which dendriticcell maturation is promoted, and antigen presentation to CD8positive T-cells induces tumour immunity (2). In addition, activation of the immune response may have an effect on cancer cells at non-irradiated sites and distant metastatic lesions (9), which is referred to as the abscopal effect (10).

The key to the technique of photoimmunotherapy is to irradiate the target lesion as accurately as possible. The quality of the pre-irradiation setting has a significant impact on the treatment outcome. Firstly, the exact extent of the target lesion should be confirmed, and the irradiation area should be set in consideration of the treatment margin. The treatment margin is set at $5-10 \mathrm{~mm}$, considering the location of large vessels. Next, the preparation for laser irradiation is performed. Strict planning is necessary to determine how to apply the laser to the area to be irradiated. Superficial lesions that can be seen can be treated relatively easily using a frontal diffuser. However, it is very difficult to insert a needle catheter into the lateral pterygoid muscle from the posterior wall of the maxillary sinus, where an ultrasound probe cannot be used, and to irradiate it with a cylindrical diffuser, as in this patient. This is because the direction for the needle catheter to puncture is unknown. The Fusion ${ }^{\mathrm{TM}}$ ENT Navigation System allowed us to place the pointer on the tumor and see the tumor extension indicated by the pointer as a dotted line in 3D CT. Accurate positioning, distance measurement, and directional confirmation were easy to achieve. Although the CT data imported for the Navigation System was from a non-contrast $\mathrm{CT}$, the lesion was confirmed by comparing it with the preoperative contrast-enhanced CT. By displaying the tumor extension as a dotted line in the contrast area of the target lesion, it was easy to confirm the direction in which the needle catheter should make a puncture. Then it was just a matter of making a puncture with the needle in that direction. The position of the internal carotid artery was also confirmed in real time, which was very useful for safety considerations.
The navigation system is a surgical support device that is frequently used in sinus surgery and does not require any special settings. In head and neck cancer of the paranasal sinuses and skull base region, there are many reports of it being used in transoral robotic-assisted skull base surgery (11). In the case of recurrent lesions in the paranasal sinuses and skull base region, we believe that the FusionTM ENT Navigation System should actively be used in combination with photoimmunotherapy, as it enables accurate puncture and safety.

\section{Conflicts of Interest}

All Authors have no conflicts of interest, potential conflicts, or financial relationships to disclose.

\section{Authors' Contributions}

Isaku Okamoto developed the case report. Isaku Okamoto wrote the main article text and prepared the figure. Isaku Okamoto, Takuro Okada, and Kunihiko Tokashiki performed the photoimmunotherapy. Isaku Okamoto, Kiyoaki Tsukahara, Takuro Okada, and Kunihiko Tokashiki oversaw the treatment of this patient. All Authors discussed the results of the case report, made comments on the article, and gave final approval of the version to be published.

\section{Acknowledgements}

The Authors would like to thank Editage (www.editage.com) for English language editing.

\section{References}

1 Mitsunaga M, Ogawa M, Kosaka N, Rosenblum LT, Choyke PL and Kobayashi H: Cancer cell-selective in vivo near infrared photoimmunotherapy targeting specific membrane molecules. Nat Med 17(12): 1685-1691, 2011. PMID: 22057348. DOI: $10.1038 / \mathrm{nm} .2554$

2 Ogawa M, Tomita Y, Nakamura Y, Lee MJ, Lee S, Tomita S, Nagaya T, Sato K, Yamauchi T, Iwai H, Kumar A, Haystead T, Shroff H, Choyke PL, Trepel JB and Kobayashi H: Immunogenic cancer cell death selectively induced by near infrared photoimmunotherapy initiates host tumor immunity. Oncotarget 8(6): 10425-10436, 2017. PMID: 28060726. DOI: 10.18632 /oncotarget.14425

3 Kobayashi H and Choyke PL: Near-infrared photoimmunotherapy of cancer. Acc Chem Res 52(8): 2332-2339, 2019. PMID: 31335117. DOI: $10.1021 /$ acs accounts.9b00273

4 Tahara M, Okano S, Enokida T, Ueda Y, Fujisawa T, Shinozaki T, Tomioka T, Okano W, Biel MA, Ishida K and Hayashi R: A phase I, single-center, open-label study of RM-1929 photoimmunotherapy in Japanese patients with recurrent head and neck squamous cell carcinoma. Int J Clin Oncol 26(10): 1812-1821, 2021. PMID: 34165660. DOI: 10.1007/s10147-021-01960-6

5 Sobin LH, Gospodarowicz MK and Wittekind C: TNM Classification of Malignant Tumours, Seventh Edition. John Wiley \& Sons, Ltd., 2009. 
6 Burduk PK, Dalke K and Kaźmierczak W: [Intraoperative navigation system in endoscopic sinus surgery]. Otolaryngol Pol 66(4 Suppl): 36-39, 2012. PMID: 23164105. DOI: 10.1016/ S0030-6657(12)70783-7

7 Sato K, Ando K, Okuyama S, Moriguchi S, Ogura T, Totoki S, Hanaoka H, Nagaya T, Kokawa R, Takakura H, Nishimura M, Hasegawa Y, Choyke PL, Ogawa $M$ and Kobayashi H: Photoinduced ligand release from a silicon phthalocyanine dye conjugated with monoclonal antibodies: a mechanism of cancer cell cytotoxicity after near-infrared photoimmunotherapy. ACS Cent Sci 4(11): 1559-1569, 2018. PMID: 30555909. DOI: 10.1021/acscentsci.8b00565

8 Nakajima K, Takakura H, Shimizu Y and Ogawa M: Changes in plasma membrane damage inducing cell death after treatment with near-infrared photoimmunotherapy. Cancer Sci 109(9): 2889-2896, 2018. PMID: 29949672. DOI: 10.1111/cas.13713

9 Nagaya T, Friedman J, Maruoka Y, Ogata F, Okuyama S, Clavijo PE, Choyke PL, Allen C and Kobayashi H: Host immunity following near-infrared photoimmunotherapy is enhanced with PD-1 checkpoint blockade to eradicate established antigenic tumors. Cancer Immunol Res 7(3): 401-413, 2019. PMID: 30683733. DOI: 10.1158/2326-6066.CIR-18-0546
10 Law AW and Mole RH: Direct and abscopal effects of $\mathrm{x}$ radiation on the thymus of the weanling rat. Int $\mathrm{J}$ Radiat Biol Relat Stud Phys Chem Med 3: 233-248, 1961. PMID: 13759646. DOI: $10.1080 / 09553006114551161$

11 Tsang RK and Chung JCK: Adapting electromagnetic navigation system for transoral robotic-assisted skull base surgery. Laryngoscope 130(8): 1922-1925, 2020. PMID: 31369158. DOI: $10.1002 /$ lary. 28220
Received January 10, 2022

Revised February 5, 2022

Accepted February 8, 2022 